\title{
Isoflurane Regulates Proliferation, Apoptosis, and Inflammatory Response of Lipopolysaccharide-Induced Human Astrocyte through the miR-206/BDNF Axis
}

\author{
Jianying Wang, Zhiyuan Liu, Xue Wang, and Yu Liu \\ Yiwu Central Hospital, Yiwu, 322000 Zhejiang, China \\ Correspondence should be addressed to Yu Liu; ycwyly@yeah.net
}

Received 31 December 2019; Accepted 14 March 2020; Published 26 March 2020

Guest Editor: Can Yang Zhang

Copyright (C) 2020 Jianying Wang et al. This is an open access article distributed under the Creative Commons Attribution License, which permits unrestricted use, distribution, and reproduction in any medium, provided the original work is properly cited.

\begin{abstract}
Objective. To investigate the effect of isoflurane (ISO) on the proliferation, apoptosis, and inflammatory response of lipopolysaccharide- (LPS-) induced normal human astrocytes (NHAs) by regulating the miR-206/BDNF axis. Methods. NHA proliferation activity was measured by MTT; NHA apoptotic rates were measured by Annexin V-FITC/PI; western blotting was used to measure the BDNF expression; ELISA was used to measure the IL-6, IL- $1 \beta$, and TNF- $\alpha$ expression in NHAs; qPCR was used to measure the expressions of miRNAs that are related to NHAs proliferation and apoptosis; dual-luciferase reporter was constructed to validate the targeting relationship between miR-206 and BDNF. Results. LPS increased the proliferation activity and decreased the apoptosis rate of NHAs which were effectively reversed by the ISO $(p<0.05)$; LPS significantly inhibited the expression of miRNAs related to proliferation and apoptosis in NHAs $(p<0.05, p<0.01)$, whereas ISO significantly increased the expression of miR-206 $(p<0.01)$ by downregulating the expression of BDNF, thus inhibiting NHA proliferation and inflammatory response and enhancing apoptosis. Conclusion. ISO can inhibit the expression of BDNF by upregulating the expression of miR-206, thereby inhibiting the proliferation and inflammatory response of NHAs and promoting its apoptosis.
\end{abstract}

\section{Introduction}

Recently, the incidence of neurodegenerative diseases such as amyotrophic lateral sclerosis (ALS) and spinal cord injury (SCI) is increasing, and the central nervous system inflammatory response is a potential mechanism for various neurodegenerative diseases [1]. It has been reported that the normal human astrocytes (NHAs) can minimize primary damage and repair damaged tissues. However, under certain pathological conditions, NHAs can change to become reactive NHAs [2], and reactive NHAs are one of the main sources of proinflammatory cytokines in the brain [3]. NHAs are involved in the development of a variety of neurodegenerative diseases [4]; therefore, studying the mechanism of reactive NHAs is helpful for the development of protective strategies for neurodegenerative diseases induced by central nervous system inflammation. Lipopolysaccharide (LPS) is the main component of Gram-negative endotoxin [5], and it is an effective immune system-activating factor, which is usually used to study inflammation-related diseases [6]. Therefore, in this study, LPS was used to treat NHAs to establish an in vitro LPS-induced NHA injury model. Isoflurane (ISO) is a commonly used inhalation anesthetic and has been shown to have neuroprotective effects by inhibiting inflammatory responses [7], but its regulatory effect on reactive NHAs has not been reported. It has been demonstrated that miR-206 was able to inhibit the inflammatory response to reduce neural pain [8] and also to regulate the proliferation and apoptosis of nerve cells [9], but the effect on NHA cells is unknown. A bioinformatics study suggests that brain-derived neurotrophic factor (BDNF) is a target protein of miR-206 and has been proved to be an important target for the treatment of neurodegenerative diseases [10]. Therefore, this study aimed at investigating the effects 
of ISO on the proliferation, apoptosis, and inflammatory response of NPS cells induced by lipopolysaccharide through the miR-206/BDNF axis.

\section{Materials and Methods}

2.1. Cell Lines and Reagents. NHAs (Cat. No. 1800) were purchased from Shanghai Fumeng Gene Biotechnology Co., Ltd. The DMEM complete medium, penicillin, streptomycin, fetal bovine serum, Lipofectamine 2000 transfection reagent, one-step reverse transcription kit, TRIzol kit, and dual-luciferase reporter gene kit were purchased from Thermo Fisher. The Annexin V-FITC/PI Apoptosis Detection Kit was purchased from Biotech Bioengineering (Shanghai) Co., Ltd. The ELISA kit was purchased from Shanghai Gaining Biotechnology Co., Ltd. RIPA cell lysates were purchased from Beijing Solaibao Technology Co., Ltd. LPS was purchased from Shanghai Guchen Biotechnology Co., Ltd. Isoflurane was purchased from Jinan Huifengda Chemical Co., Ltd. BDNF primary and secondary antibodies were purchased from Wuhan Aimeijie Technology Co., Ltd.

2.2. Cell Culture. NHAs were cultured and incubated in the DMEM medium containing 10\% fetal bovine serum, $1 \%$ penicillin, and streptomycin and cultured in a $37^{\circ} \mathrm{C}, 5 \% \mathrm{CO}_{2}$ incubator. The culture medium was replaced every day. The passage was performed at a confluency of 70 to $80 \%$, and cells from passages 3 to 4 were used for subsequent experiments.

Drug treatment. NHAs in logarithmic growth phase were taken and treated with different concentrations of LPS $(0 \mu \mathrm{g} / \mathrm{mL}, 0.2 \mu \mathrm{g} / \mathrm{mL}, 0.5 \mu \mathrm{g} / \mathrm{mL}$, and $1 \mu \mathrm{g} / \mathrm{mL})$ for $24 \mathrm{~h}$, and the appropriated LPS concentration was selected. After $24 \mathrm{~h}$ of treatment with LPS $(0.5 \mu \mathrm{g} / \mathrm{mL})$, the NHAs were placed in a closed anesthesia box. The inlet end was connected to an ISO gas volatilizer, and $95 \%$ air and $5 \% \mathrm{CO}_{2}$ were passed in. The outlet end was connected to an anesthetic gas monitor for the ISO concentration examination. Different concentrations of ISO $(0.7 \%, 1.4 \%$, and $2.1 \%)$ were passed in for $2 \mathrm{~h}$, and then cell proliferation, apoptosis, and inflammatory factors were measured.

2.3. Cell Transfection. NHAs in the logarithmic growth phase were taken, inoculated into 6-well plates, and cultured in a $37^{\circ} \mathrm{C}, 5 \% \mathrm{CO}_{2}$ incubator. When the confluence of the cells reaches 70 80\%, miR-206 mimics, anti-miR-206, NCmimics, and si-BDNF were transfected into NHAs and cultured in a $5 \% \mathrm{CO}_{2}$ incubator at $37^{\circ} \mathrm{C}$ for $48 \mathrm{~h}$ before use.

2.4. NHA Proliferation Activity Measured by MTT. Each group of NHAs to be tested in the logarithmic growth phase was taken and inoculated in a 96-well plate at a density of $1 \times 10^{4}$ cells/well. $10 \mu \mathrm{L}$ MTT solution was subsequently added when the cells cultured for $0,24,48,72$, and $96 \mathrm{~h}$. After a further $4 \mathrm{~h}$ of incubation, the culture supernatant was aspirated, and $100 \mu \mathrm{L}$ of dimethyl sulfoxide (DMSO) was added; finally, the absorbance (OD) was measured at $450 \mathrm{~nm}$ using a microplate reader.

2.5. NHA Apoptotic Rates Detected by Annexin V-FITC/PI Double Staining. NHAs to be tested in each group were col-
TABLE 1: qPCR primer sequences.

\begin{tabular}{lr}
\hline Primer & Sequence $\left(5^{\prime}\right.$ - $\left.3^{\prime}\right)$; F: forward primer, R: reversed primer \\
\hline miR-211 & F: $5^{\prime}$-TTGTGGGCTTCCCTTTGTCATCCT-3' \\
R: $5^{\prime}$-TGCTGTGGGAAGTGACAACTGA-3' \\
FiR-146a & F: $5^{\prime}$-CCTGAGAAGTGAATTCCATGGG-3' \\
& R: $5^{\prime}$-TGGTGTCGTGGAGTCG-3' \\
miR-140 & F: $5^{\prime}$-CCCAAGCTTTTTCCGTGGTGACCTCCTCT-3' \\
& R: $5^{\prime}$-CGCGGATCCTGCTGGGCTGTTTGTGGGGG-3' \\
miR-206 & F: $5^{\prime}$-CGGGCTTGTGGAATGGTAAGC-3' \\
R6 $5^{\prime}$-GCTTCGGCAGCACATATACTAAAAT-3' & F: $5^{\prime}$-CTCGCTTCGGCAGCACA-3' \\
R: $5^{\prime}$-AACGCTTCACGAATTTGCGT-3'
\end{tabular}

lected and cultured for $48 \mathrm{~h}$ and digested with $0.25 \%$ trypsin and centrifuged at $1000 \mathrm{r} / \mathrm{min}$ for $5 \mathrm{~min}$. After that, the cells were adjusted to a concentration of $5 \times 10^{5}$ cells $/ \mathrm{mL}$, and $5 \mu \mathrm{L}$ Annexin V-FITC was added and mixed well. After homogenization, the cells were incubated at room temperature for $15 \mathrm{~min}$ in the dark, then $5 \mu \mathrm{L}$ of PI staining solution was added, mixed, and incubated for $5 \mathrm{~min}$; and finally, flow cytometry was used to detect the apoptosis level of NHAs.

2.6. Expression of $I L-6, I L-1 \beta$, and TNF- $\alpha$ in NHAs Determined by ELISA. Each group of NHAs to be tested was collected and prepared into a cell suspension, and the expression of IL-6, IL- $1 \beta$, and TNF- $\alpha$ in NHAs was determined according to the corresponding ELISA kit instructions.

2.7. miRNAs Expression in NHAs Measured by qPCR. The TRIzol method was used to extract the total RNA, which was then reverse transcribed into cDNA according to the instructions of the miRNA reverse transcription kit. qPCR was used according to the instructions on the miRNA qPCR kit. U6 was used as an internal reference, and the primer sequences are shown in Table 1 . The relative expressions were calculated using $2^{-\Delta \Delta \mathrm{Ct}}$.

2.8. BDNF Expression in NHAs Detected by Western Blotting. NHAs of each group in the logarithmic growth phase were taken and washed twice with PBS. A total of $0.5 \mu \mathrm{g} / \mathrm{mL}$ LPS were added to the experimental group, and cells were rinsed with PBS twice after $24 \mathrm{~h}$ of culture. After that, $1 \mathrm{~mL}$ of PBS was added before centrifugation $(3000 \mathrm{r} / \mathrm{min})$ for $5 \mathrm{~min}$. After centrifugation, the supernatant was discarded, and the cells were washed three times by adding icy PBS before the addition of the RIPA lysis buffer. The BCA protein quantification kit was used to determine the protein concentration. An equal amount of protein samples was then taken from each group for the SDS-PAGE electrophoresis. After electrophoresis, the SDS-PAGE gel was transferred to a PVDF membrane and placed in a 5\% skim milk powder for blockage for $1.5 \mathrm{~h}$. A primary antibody $(1: 1000)$ was added and incubated at $4^{\circ} \mathrm{C}$ overnight. On the next day, after washing the membrane once with TBST, an HRP-labeled secondary 


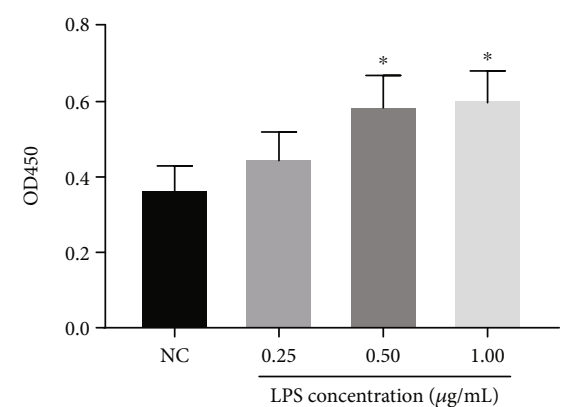

(a)

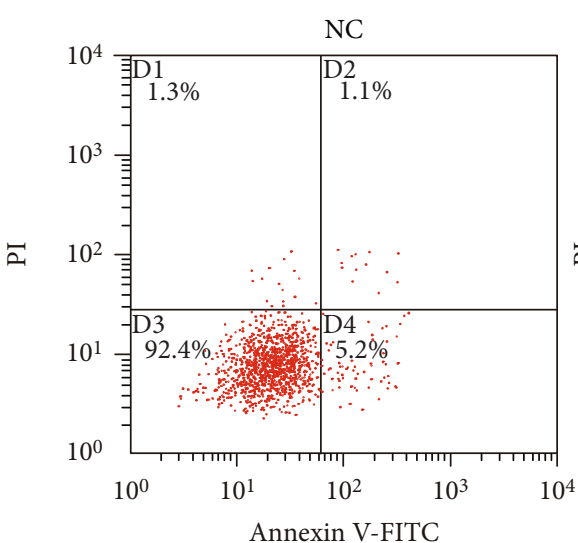

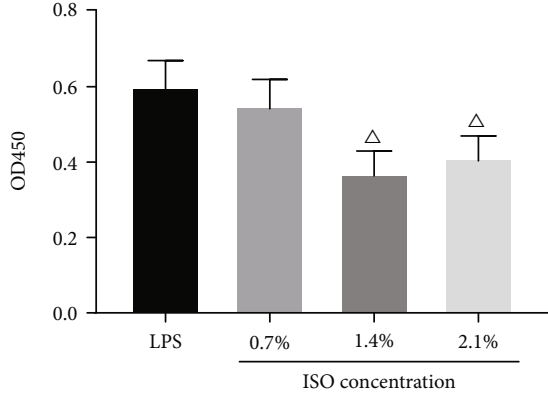

(b)

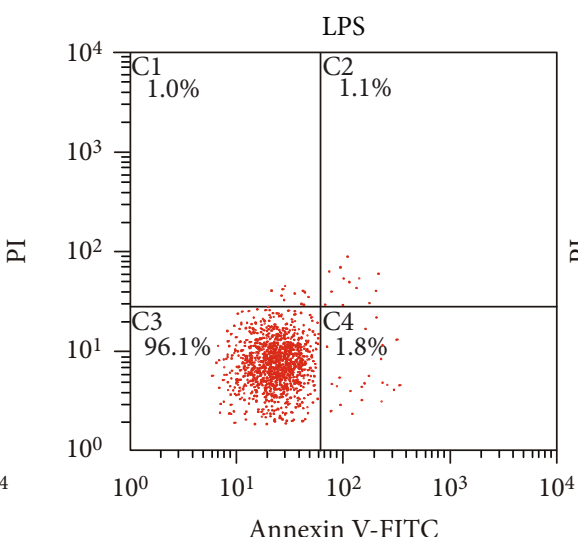

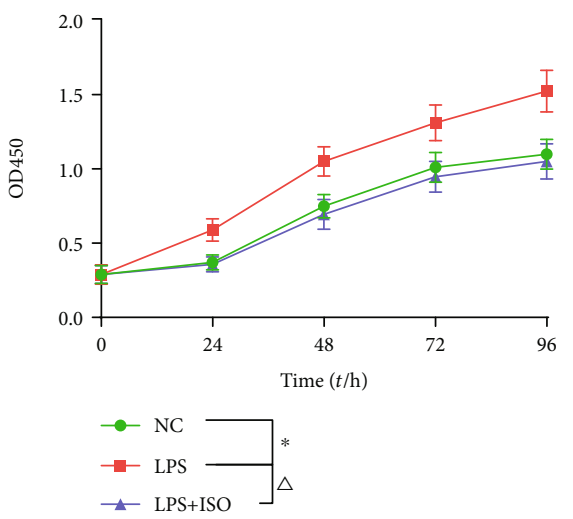

(c)

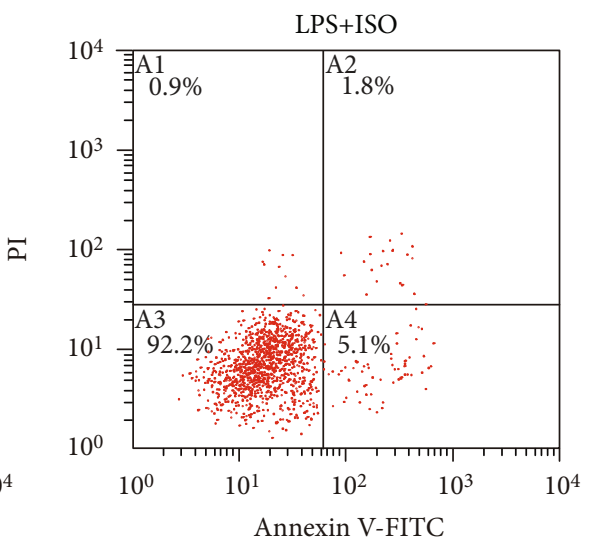

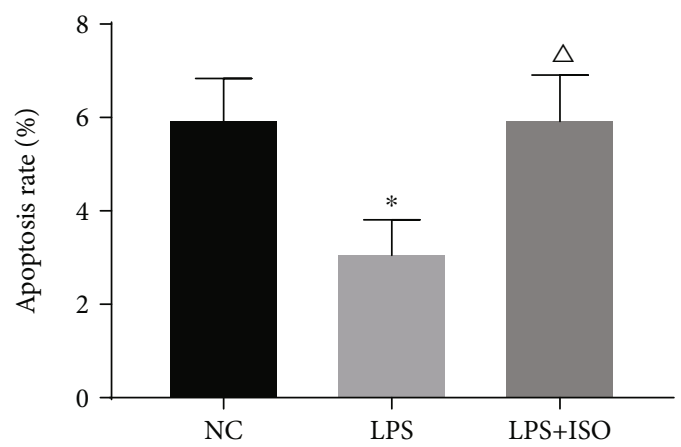

(d)

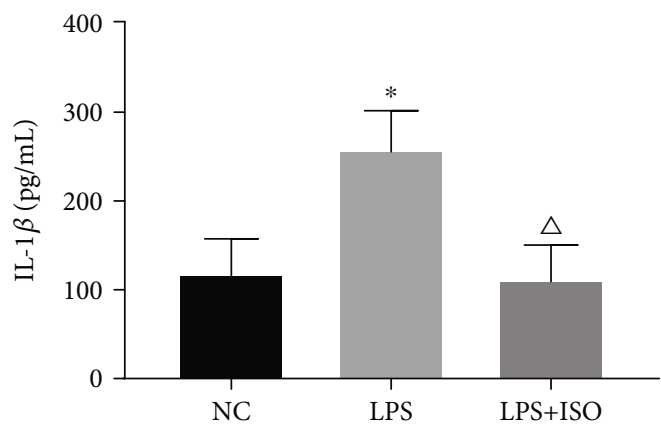

(e)

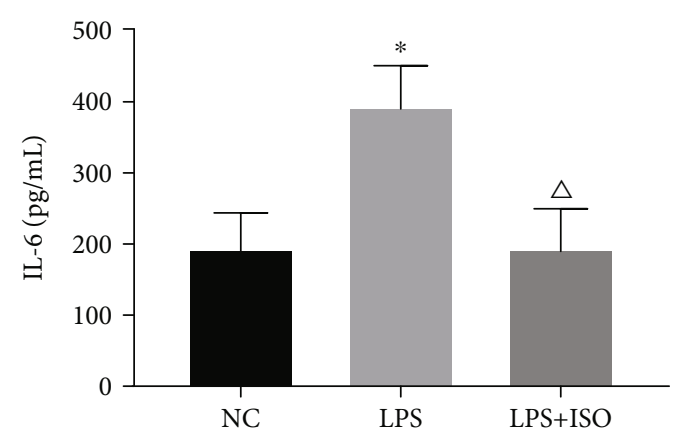

(f)

Figure 1: Continued. 


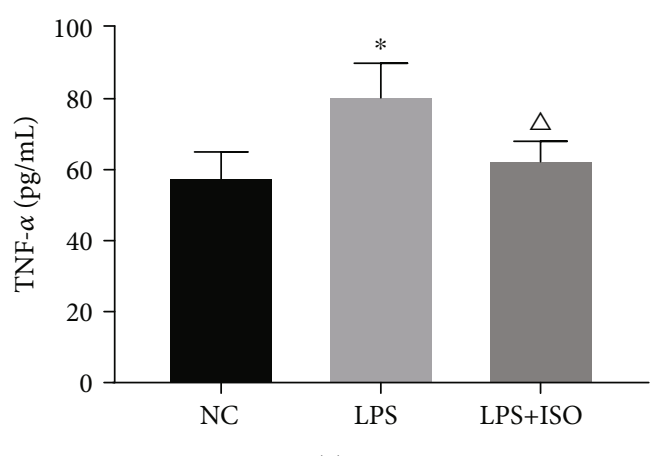

(g)

FIGURE 1: Effects of ISO on LPS-induced NHAs proliferation, apoptosis, and inflammatory response. ${ }^{*} p<0.05$ vs. the $N C$ group; ${ }^{\Delta} p<0.05$ vs. the LPS group. (a-c) The proliferation of NHAs measured by MTT. (d) NHA apoptosis measured by Annexin V-FITC/PI. (e-g) Expression of IL-6, IL- $1 \beta$, and TNF- $\alpha$ in NHAs determined by ELISA.

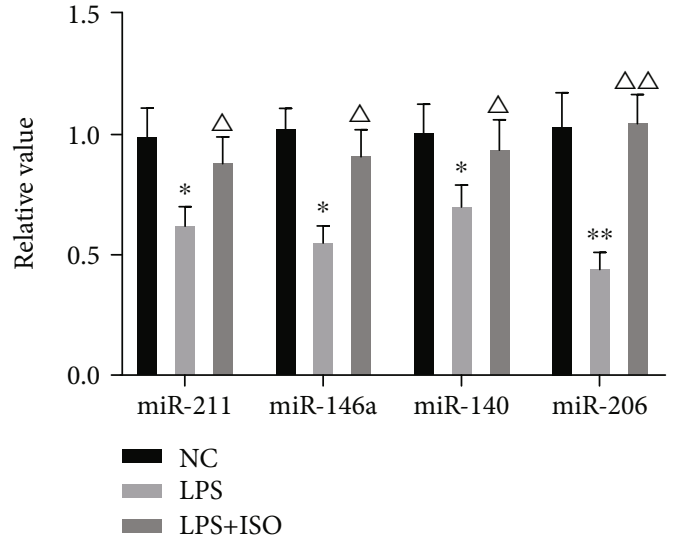

FIGURE 2: ISO upregulated the expression of miR-206 in NHAs cells. ${ }^{*} p<0.05,{ }^{* *} p<0.01$ vs. the NC group; ${ }^{\Delta} p<0.05,{ }^{\Delta \Delta} p<0.01$ vs. the LPS group.

antibody (1:5000) was added to the sample and incubated for $1.5 \mathrm{~h}$ at room temperature. After that, the membrane was washed three times with TBST, and the ECL kit was used for the development of the protein bands. The ImageJ software was then used to analyze the grayscale of the bands and calculate the relative expression.

2.9. Targeting the Relationship between miR-206 and BDNF Verified by Dual-Luciferase Reporter. The StarBase database was used to predict the binding site of miR-206 and BDNF. The BDNF wild-type vector (WT-BDNF) was constructed; gene mutation technology was used to change the binding site of miR-206 and BDNF, and the BDNF mutant vector (MUT-BDNF) was constructed by the same method. WTBDNF or MUT-BDNF was then cotransfected with miR206 mimics or miR-NC into NHAs and cultured for $48 \mathrm{~h}$, and the luciferase activity of each group was measured using the dual-luciferase reporter gene kit [8].

2.10. Statistical Analysis. All experiments in this study were repeated three times, and data were expressed as $\bar{x} \pm s$. The GraphPad 7.0 software was used for statistical analysis and related figure drawing. $t$-test was used for comparison between two groups, and one-way analysis of variance was used for comparison between multiple groups. $p<0.05$ and $p<0.01$ were used to indicate a statistically significant difference.

\section{Results}

3.1. Effect of ISO on LPS-Induced NHAs Proliferation, Apoptosis, and Inflammatory Response. MTT results showed that the cell proliferation ability increased in a dosedependent manner after treatment of NHAs with different concentrations of LPS $(0.0 \mu \mathrm{g} / \mathrm{mL}, 0.2 \mu \mathrm{g} / \mathrm{mL}, 0.5 \mu \mathrm{g} / \mathrm{mL}$, and $1.0 \mu \mathrm{g} / \mathrm{mL}$ ) (Figure $1(\mathrm{a}), p<0.01$ ), and there is no significant difference in cell proliferation activity when LPS was at a concentration of $0.5 \mu \mathrm{g} / \mathrm{mL}$ and $1 \mu \mathrm{g} / \mathrm{mL}$. After NHAs were first treated with $0.5 \mu \mathrm{g} / \mathrm{mL}$ LPS, different concentrations of ISO $(0.7 \%, 1.4 \%$, and $2.1 \%)$ were introduced, and the proliferation activity of NHAs was found to be the lowest at $1.4 \%$ after $2 \mathrm{~h}$ (Figure 1(b), $p<0.01$ ). MTT, Annexin V-FITC/PI, and ELISA were used to measure the proliferation activity, apoptosis rate, IL- $6, \mathrm{IL}-1 \beta$, and TNF- $\alpha$ expression of NHAs in the NC group, LPS group, and LPS+ISO group. The results showed that compared with the NC group, the proliferation activity and the expression of IL- 6 , IL- $1 \beta$, and TNF- $\alpha$ were significantly increased, and the apoptosis rate was significantly reduced in the LPS group, whereas the LPS+ISO group reversed the effects of the LPS on NHA proliferation, apoptosis, and inflammatory response (Figures 1(c)-1(e), all $p<$ 0.05). Therefore, taken together, ISO can reverse the promotion effect of LPS on the proliferation and inflammatory response of NHAs and the inhibitory effect on apoptosis.

3.2. ISO Upregulated miR-206 Expression in NHAs. qPCR results showed that LPS significantly inhibited the expression of miRNAs [2, 11-13] associated with proliferation and apoptosis of NHAs $(p<0.05, p<0.01)$, and the addition of ISO reversed these inhibitory effects to a certain extent. In particular, among all examined miRNAs, miR-206 was the most significantly reversed one (Figure 2, $p<0.01$ ). Therefore, it can be concluded that ISO upregulated the expression of miR-206 in NHAs.

3.3. miR-206 Downregulated BDNF. The bioinformatics database StarBase was used to predict that BDNF is a potential 

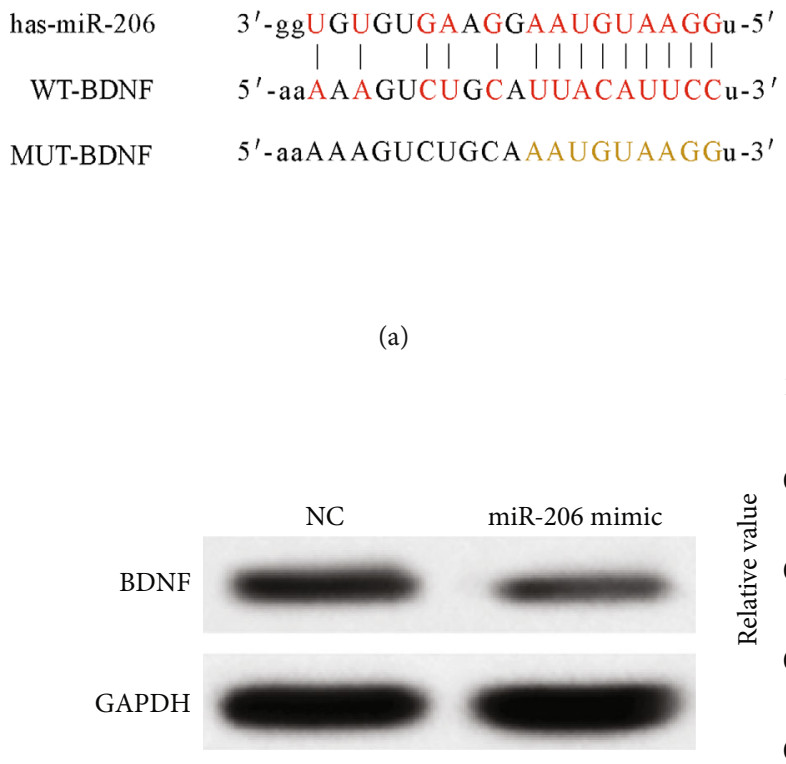

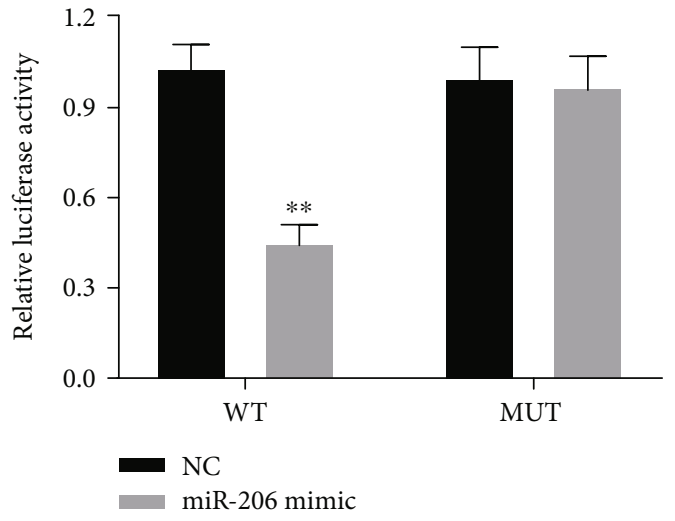

(b)

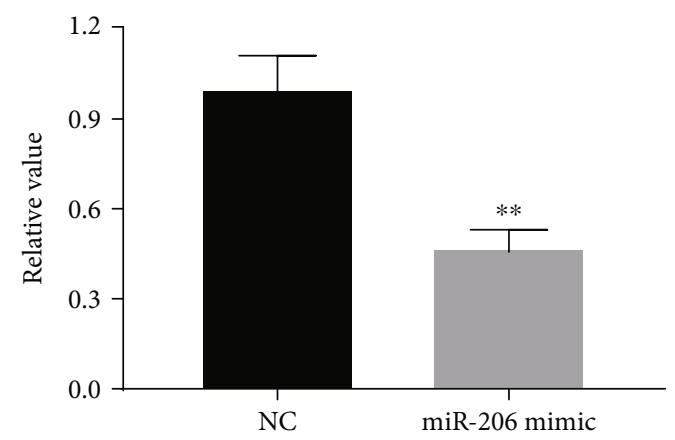

(c)

FIGURE 3: miR-206 targeted downregulation of BDNF. (a) The bioinformatics analysis result showed that BDNF was a target gene of miR-206. (b) The dual-luciferase reporter gene assay was used to verify the relationship between miR-206 and BDNF. (c) Western blotting was applied to measure the expression of BDNF. ${ }^{* *} p<0.01$ vs. the $N C$ group.

target gene for miR-206 (Figure 3(a)). The results of the dual-luciferase reporter gene showed that the overexpression of miR-206 significantly reduced the luciferase activity of the WT-BDNF plasmid $(p<0.01)$; however, it had no significant effect on the luciferase activity of the MUT-BDNF plasmid $(p>0.05)$. Western blotting results (Figure 3(c)) also confirmed that the overexpression of miR-206 significantly inhibited BDNF expression in NHAs $(p<0.01)$. These results suggested that miR-206 targeted and downregulated BDNF expression.

3.4. ISO Regulated the LPS-Induced Proliferation, Apoptosis, and Inflammatory Response of NHAs via the miR-206/BDNF Axis. Western blotting was used to detect BDNF expression in NHAs after treatment with LPS, and the results showed that after knocking down BDNF, BDNF expression in NHAs was significantly reduced $(p<0.01)$; in addition, after simultaneous knockdown of BDNF and miR-206, BDNF expression was significantly increased compared with knockdown of BDNF alone $(p<0.01)$, and there was no significant difference compared with the NC group. After NHAs were treated with LPS, MTT, Annexin V-FITC/PI, and ELISA were used to detect the proliferation activity, apoptosis rate, and IL-6 IL- $1 \beta$ expression of NHAs in NC group, ISO group, ISO +anti-miR-206 group, ISO+si-BDNF group, and ISO+antimiR-206+si-BDNF group. The results showed that compared with the NC group, the proliferation activity, IL-6, IL- $1 \beta$, and
TNF- $\alpha$ expression of NHAs in ISO group were significantly reduced, while the apoptosis rate increased significantly, whereas addition of anti-miR-206 effectively reversed the effects of the ISO on NHAs proliferation, apoptosis, and inflammatory response. However, in the ISO+si-BDNF group, the effects of the ISO group on NHAs proliferation, apoptosis, and inflammatory responses were further heightened. Compared with the ISO+anti-miR-206 group, the proliferation activity and inflammatory response of NHAs cells in the ISO+anti-miR-206+si-BDNF group were reduced, while the apoptosis rate was increased.

Compared with the ISO+si-BDNF group, the proliferation activity and inflammatory response of NHAs in the ISO+anti-miR-206+si-BDNF group increased, whereas the apoptosis rate decreased as shown in Figures 4(b)-4(d) (all $p<0.05$ ). Taken all these together, it seems that ISO can reverse the effects of LPS on NHAs including the promotion of proliferation, inflammatory response, and inhibition of apoptosis by regulating the miR-206/BDNF axis.

\section{Discussion}

Normal human NHAs are the most abundant cell types in the central nervous system, providing nutritional support for neurons and maintaining the function of the central nervous system [14]. Studies have shown that the function of NHAs is regulated by microglia, microorganisms, and 

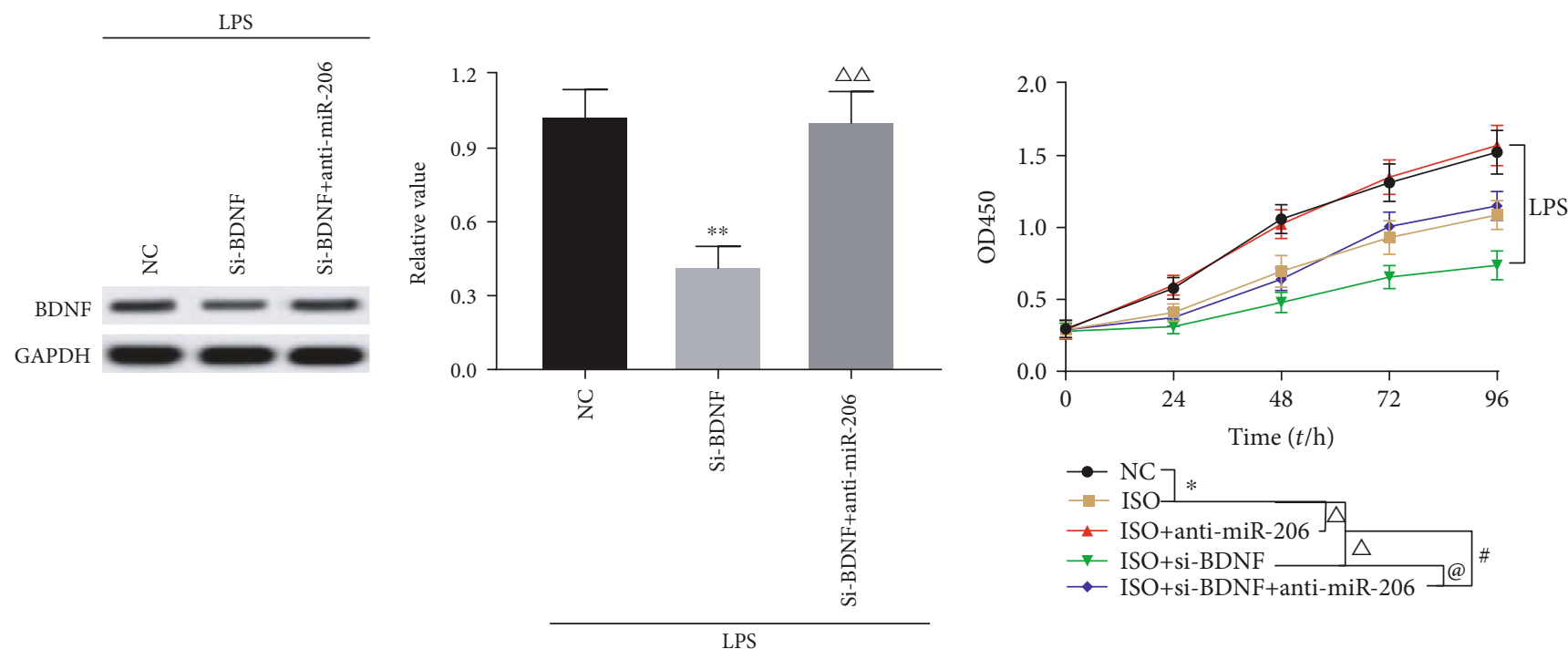

(a)

(b)
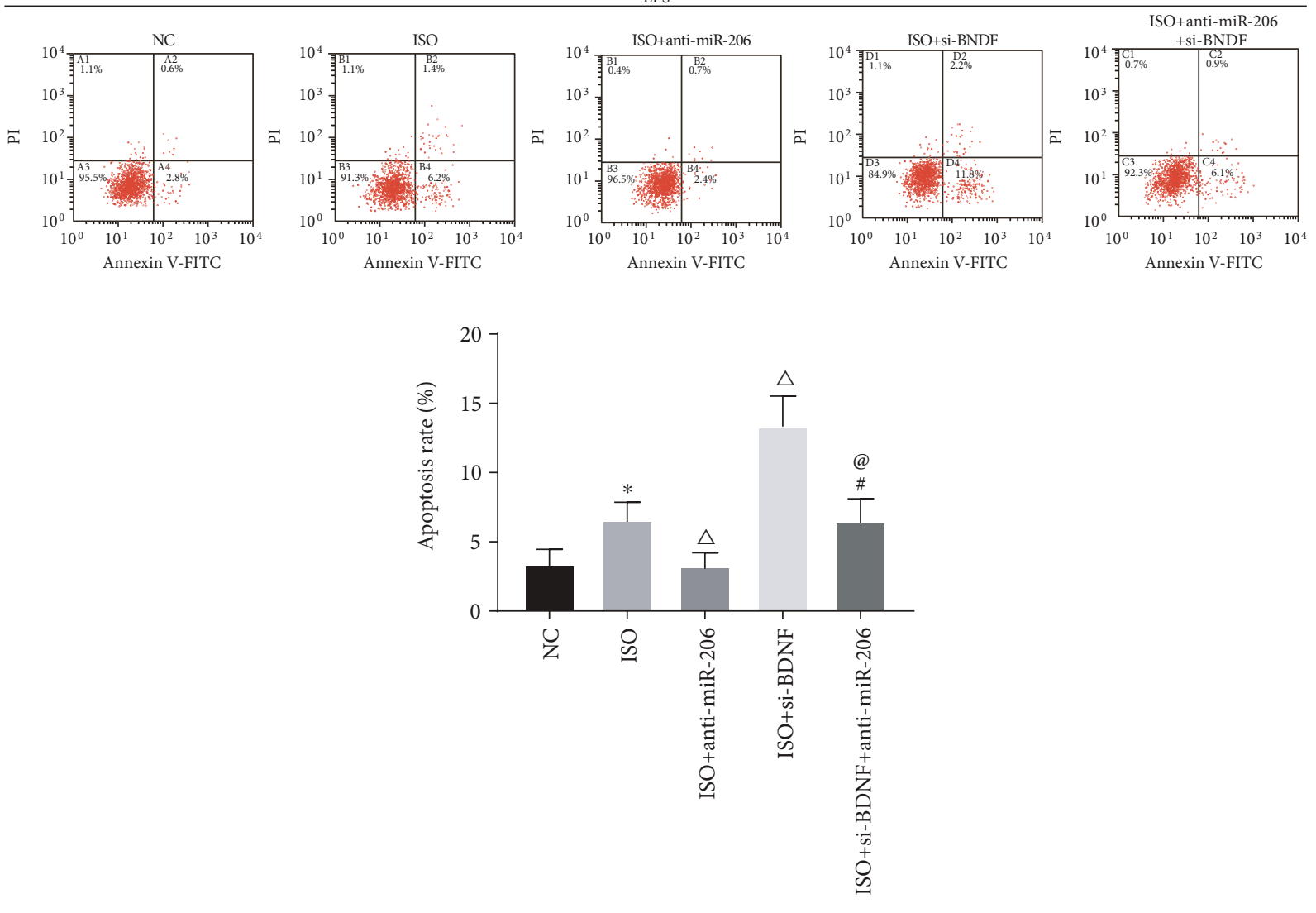

LPS

(c)

Figure 4: Continued. 


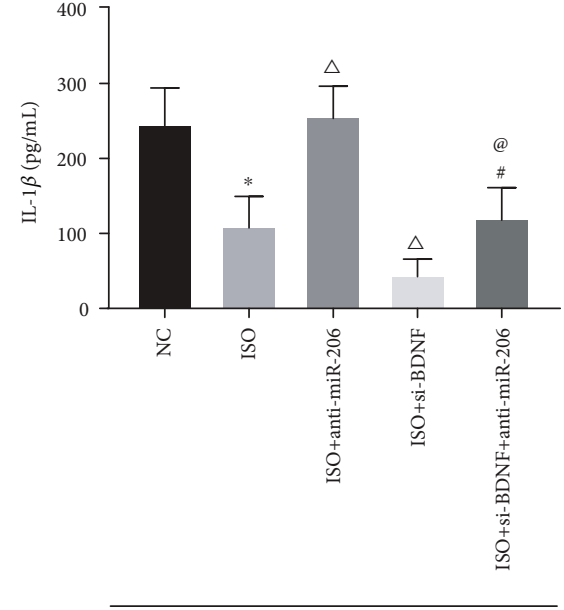

(d)

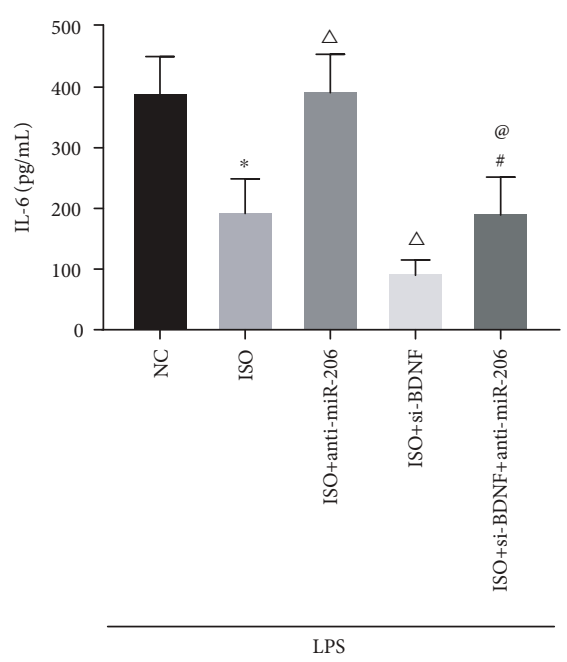

(e)

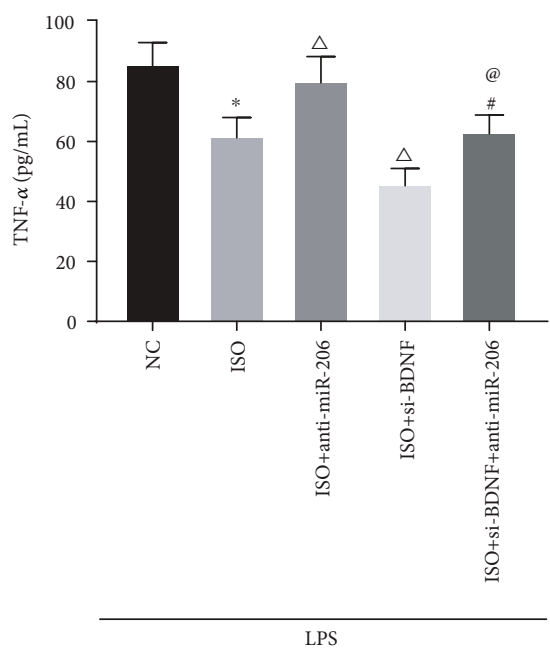

(f)

FIGURE 4: ISO regulated the effects of miR-206/BDNF axis on LPS-induced NHA cell proliferation, apoptosis, and inflammatory response. (a) Western blotting was applied to measure the expression of BDNF. (b) The proliferation activity of NHAs cells was detected by MTT. (c) Annexin V-FITC/PI was used to detect NHA cell apoptosis. (d-f) Expression of IL-6, IL-1 $\beta$, and TNF- $\alpha$ in NHAs cells was detected by ELISA. ${ }^{*} p<0.05$ vs. the NC group; ${ }^{\Delta} p<0.05$ vs. the ISO group; ${ }^{*} p<0.05$ vs. the $I S O+$ anti-miR-206; ${ }^{\circledR} p<0.05$ vs. the ISO+si-BDNF.

the internal environment [15], and under the stimulation of the inflammatory environment, NHAs can lose most of the normal astrocyte functions and gain new neurotoxic function [16]. It has also been reported that NHA cellderived inflammation is a common component of acute or chronic damage to the central nervous system [17] and plays a key regulatory role in the pathogenesis of various neurodegeneration caused by neuroinflammation [18]. The results in our study found that LPS can promote the proliferation and inflammatory response of NHA cells and inhibit its apoptosis.

ISO is a commonly used inhalation anesthetic and is widely used in various types of surgery to maintain general anesthesia. In addition, ISO also has anti-inflammatory, antioxidant, and apoptotic effects [1]. It has been reported that ISO can regulate immune and inflammatory responses by reducing neutrophil function and inhibiting cytokines release by macrophages and attenuating the response of natural killer cells to interferon- $\alpha 4$, finally to exert anti-inflammatory effects by reducing systemic inflammation and inhibiting cytokines release by mononuclear cells and macrophages release [19]. Moreover, studies have demonstrated that ISO was able to protect brain damage. For instance, Swissa [7] and others found that ISO significantly reduced the late-stage brain damage in the rat model of epilepsy status induced by oxophosphate. However, it has not yet been reported whether ISO regulates reactive NHA cell-derived inflammation. Our results discovered that ISO can inhibit LPS-induced proliferation and inflammatory response and promote apoptosis of NHAs cells.

miR-206 has been shown associated with neuronal activation and neuroinflammation [20] and is abnormally lowly expressed in the serum of chronic neuropathy rats [21]. In addition, miR-206 can regulate inflammatory responses. For example, overexpression of miR-206 can reduce neuro- pathic pain caused by overexpression of ZEB2 in microglia in rats by inhibiting neuroinflammation [22]. BDNF is produced and secreted by neurons, it can promote the development of axons in an autocrine manner, and it is essential in the development of brain circuits [3]. In addition, BDNF, as a neurotrophic protein, plays an important role in the plasticity of the central nervous system and is also involved in the pathogenesis of neurological diseases [23], thus becoming an important target for the treatment of neurodegenerative diseases. The results in this study illustrated that ISO can upregulate miR-206 expression, and miR206 can inhibit LPS-induced glial cell proliferation, inflammatory, and promote its apoptosis through downregulation of BDNF expression.

In summary, ISO can reduce the inflammatory response under pathological conditions by upregulating the inhibitory effect of miR-206, inhibiting LPS-induced NHAs proliferation, and enhancing apoptosis.

\section{Data Availability}

All the data is available with the handwritten notebook documented in our lab.

\section{Conflicts of Interest}

The authors declare that there are no conflicts of interest regarding the publication of this paper.

\section{Acknowledgments}

This work is supported by the Zhejiang Science and Technology Department Project (No. 2013C33216). 


\section{References}

[1] S. Yang, J. Liu, X. Zhang et al., "Anesthetic isoflurane attenuates activated microglial cytokine-induced VSC4.1 motoneuronal apoptosis," American Journal of Translational Research, vol. 8, no. 3, pp. 1437-1446, 2016.

[2] K. Zhang, S. Wu, Z. Li, and J. Zhou, "MicroRNA-211/BDNF axis regulates LPS-induced proliferation of normal human astrocyte through PI3K/AKT pathway," Bioscience Reports, vol. 37, no. 4, 2017.

[3] T. Tanaka, S. Kai, T. Matsuyama, T. Adachi, K. Fukuda, and K. Hirota, "General anesthetics inhibit LPS-induced IL-1beta expression in glial cells," PLoS One, vol. 8, no. 12, article e82930, 2013.

[4] A. Birger, I. Ben-Dor, M. Ottolenghi et al., "Human iPSCderived astrocytes from ALS patients with mutated C9ORF72 show increased oxidative stress and neurotoxicity," EBioMedicine, vol. 50, pp. 274-289, 2019.

[5] Y. Ding, J. Zhang, and R. Wang, "Inhibition of tissue transglutaminase attenuates lipopolysaccharide-induced inflammation in glial cells through AKT/mTOR signal pathway," Biomedicine \& Pharmacotherapy, vol. 89, pp. 1310-1319, 2017.

[6] X. Tao, M. Yan, L. Wang et al., "Homeostasis imbalance of microglia and astrocytes leads to alteration in the metabolites of the kynurenine pathway in LPS-induced depressive-like mice," International Journal of Molecular Sciences, vol. 21, no. 4, p. 1460, 2020.

[7] E. Swissa, G. Bar-Klein, Y. Serlin et al., "Midazolam and isoflurane combination reduces late brain damage in the paraoxon-induced status epilepticus rat model," Neurotoxicology, vol. 78, pp. 99-105, 2020.

[8] W. Sun, L. Zhang, and R. Li, "Overexpression of miR-206 ameliorates chronic constriction injury-induced neuropathic pain in rats via the MEK/ERK pathway by targeting brainderived neurotrophic factor," Neuroscience Letters, vol. 646, pp. 68-74, 2017.

[9] R. Wang, Y. Hu, G. Song et al., "MiR-206 regulates neural cell proliferation and apoptosis via Otx2," Cellular Physiology and Biochemistry, vol. 29, no. 3-4, pp. 381-390, 2012.

[10] C. Deng, J. Zhu, J. Yuan, Y. Xiang, and L. Dai, "Pramipexole inhibits MPP (+)-induced neurotoxicity by miR-4943p/BDNF," Neurochemical Research, vol. 45, no. 2, pp. 268$277,2019$.

[11] J. Mei, R. Bachoo, and C. L. Zhang, "MicroRNA-146a inhibits glioma development by targeting Notch1," Molecular and Cellular Biology, vol. 31, no. 17, pp. 3584-3592, 2011.

[12] Z. Tu, Y. Li, Y. Dai et al., "MiR-140/BDNF axis regulates normal human astrocyte proliferation and LPS-induced IL-6 and TNF-alpha secretion," Biomedicine \& Pharmacotherapy, vol. 91, pp. 899-905, 2017.

[13] T. J. Liu, B. Wang, Q. X. Li, X. L. Dong, X. L. Han, and S. B. Zhang, "Effects of microRNA-206 and its target gene IGF-1 on sevoflurane-induced activation of hippocampal astrocytes in aged rats through the PI3K/AKT/CREB signaling pathway," Journal of Cellular Physiology, vol. 233, no. 5, pp. 4294-4306, 2018.

[14] L. Udovin, C. Quarracino, M. I. Herrera, F. Capani, M. OteroLosada, and S. Perez-Lloret, "Role of Astrocytic Dysfunction in the Pathogenesis of Parkinson's Disease Animal Models from a Molecular Signaling Perspective," Neural Plasticity, vol. 2020, Article ID 1859431, 10 pages, 2020.
[15] M. A. Wheeler, I. C. Clark, E. C. Tjon et al., "MAFG-driven astrocytes promote CNS inflammation," Nature, vol. 578, no. 7796, pp. 593-599, 2020.

[16] S. A. Liddelow, K. A. Guttenplan, L. E. Clarke et al., "Neurotoxic reactive astrocytes are induced by activated microglia," Nature, vol. 541, no. 7638, pp. 481-487, 2017.

[17] X. Duan, A. Zohaib, Y. Li et al., "miR-206 modulates lipopolysaccharide-mediated inflammatory cytokine production in human astrocytes," Cellular Signalling, vol. 27, no. 1, pp. 61-68, 2015.

[18] A. Montoya, D. Elgueta, J. Campos et al., "Dopamine receptor D3 signalling in astrocytes promotes neuroinflammation," Journal of Neuroinflammation, vol. 16, no. 1, p. 258, 2019.

[19] X. Guo, J. Deng, B. Zheng et al., "HDAC1 and HDAC2 regulate anti-inflammatory effects of anesthetic isoflurane in human monocytes," Immunology and Cell Biology, 2020.

[20] M. Kashyap, S. Pore, M. Chancellor, N. Yoshimura, and P. Tyagi, "Bladder overactivity involves overexpression of MicroRNA 132 and nerve growth factor," Life Sciences, vol. 167, pp. 98-104, 2016.

[21] Y. Xu, X. Zhang, S. Pu, J. Wu, Y. Lv, and D. du, "Circulating microRNA expression profile: a novel potential predictor for chronic nervous lesions," Acta Biochimica et Biophysica Sinica, vol. 46, no. 11, pp. 942-949, 2014.

[22] Z. L. Chen, J. Y. Liu, F. Wang, and X. Jing, "Suppression of MALAT1 ameliorates chronic constriction injury-induced neuropathic pain in rats via modulating miR-206 and ZEB2," Journal of Cellular Physiology, vol. 234, no. 9, pp. 1564715653, 2019.

[23] G. Cavaliere, G. Trinchese, E. Penna et al., "High-fat diet induces neuroinflammation and mitochondrial impairment in mice cerebral cortex and synaptic fraction," Frontiers in Cellular Neuroscience, vol. 13, p. 509, 2019. 